\title{
Estudo do Fenômeno da Ilha de Calor na Cidade de Manaus/AM: Um Estudo a Partir de Dados de Sensoriamento Remoto, Modelagem e Estações Meteorológicas
}

\author{
Polari Batista Corrêa ${ }^{1}$, Luiz Antonio Candido ${ }^{2}$, Rodrigo Augusto Ferreira de Souza ${ }^{3}$, \\ Rita Valéria Andreoli ${ }^{3}$, Mary Toshie Kayano ${ }^{4}$ \\ ${ }^{1}$ Programa de Pós-Graduação em Clima e Ambiente, \\ Instituto Nacional de Pesquisas da Amazônia, Manaus, AM, Brasil. \\ ${ }^{2}$ Instituto Nacional de Pesquisas da Amazônia, Manaus, AM, Brazil. \\ ${ }^{3}$ Escola Superior de Tecnologia, Universidade do Estado do Amazonas, Manaus, AM, Brasil. \\ ${ }^{4}$ Centro de Previsão de Tempo e Estudos Climáticos, Instituto Nacional de Pesquisas Espaciais, \\ São José dos Campos, SP, Brasil.
}

Recebido: 29/1/2015 - Aceito: 8/9/2015

\begin{abstract}
Resumo
O fenômeno da ilha de calor (IC) na cidade de Manaus foi estudado usando dados de modelagem do clima urbano com o modelo Weather Research and Forecasting (WRF) e dados de estações meteorológicas. Foram usados os dados de temperatura da superfície (TS) obtidos pelo sensor MODIS do satélite ambiental Aqua, produto MYD11A2 na resolução horizontal de $1 \mathrm{~km}$ por um $1 \mathrm{~km}$ para o período de 2002 a 2012 na cidade de Manaus para avaliar o processo de urbanização. Foi realizado um estudo de caso com as simulações de TS e de temperatura do ar (TA) nos meses de agosto e setembro de 2009 que foram comparadas, respectivamente, com os dados de satélite e de estações meteorológicas. Identificou-se a distribuição espacial da TS na cidade, e as diferenças de TS entre a área urbana e a de floresta e entre a área urbana e de rios foram quantificadas. As diferenças de TS ao longo das faixas de longitude de $60,03^{\circ} \mathrm{O}$ a $59,97^{\circ} \mathrm{O}$ e de latitude de $3,09^{\circ} \mathrm{S} \mathrm{a}-3,03^{\circ} \mathrm{S}$ foram analisadas com os dados do modelo WRF e do sensor MODIS. A intensidade da IC estimada com os dados de TA do modelo e de estações meteorológicas foram, em média, respectivamente de $1,87^{\circ} \mathrm{C}$ e de $1,80^{\circ} \mathrm{C}$, consistentes com resultados anteriores. Portanto, a TA simulada pode ser usada para fins de estimar a intensidade da IC em Manaus.
\end{abstract}

Palavras-chave: clima urbano, sensor MODIS, modelo WRF, temperatura da superfície.

\section{Heat Islands in Manaus City: Study with Remote Sensing Data, Modeling and Meteorological Data}

\begin{abstract}
The heat island phenomenon (HI) in the city of Manaus was studied using the urban climate modeling with the Weather Research and Forecasting (WRF) model and meteorological weather station data. The surface temperature (ST) data from the environmental satellite MODIS Aqua, product MYD11A2 with a horizontal resolution of $1 \mathrm{~km}$ by $1 \mathrm{~km}$ for the period 2002 to 2012 were used to evaluate the urbanization process in Manaus city. A case study was conducted with the simulated ST and air temperature (AT) for the months of August and September 2009, which were compared respectively with the satellite data and meteorological weather station data. The spatial distribution of ST in the city was identified, and the ST differences between the urban and the forest areas and between the urban and river areas were quantified. The ST difference across the longitude bands $60,03^{\circ} \mathrm{W}$ to $59,97^{\circ} \mathrm{W}$ and across the latitude bands $3,09^{\circ} \mathrm{S}$ a $-3,03^{\circ} \mathrm{S}$ were analyzed with WRF data and MODIS data. The intensity of the HI estimated with the AT from the model and the meteorological stations were on average respectively of $1,87^{\circ} \mathrm{C}$ and $1,80^{\circ} \mathrm{C}$, consistent with previous results. Therefore, the simulated AT can be used to estimate the intensity ofHI in Manaus.
\end{abstract}

Keywords: climate urban, MODIS sensor, WRF model, surface temperature.

Autor de correspondência: Polari Batista Corrêa, e-mail: polaribc@gmail.com. 


\section{Introdução}

Atualmente, mais de $50 \%$ da população mundial vive em cidades e espera-se que esse percentual chegue a $81 \%$ em 2030 (UNFPA, 2007). O processo de urbanização global acelerou-se tanto em intensidade como em área física, o que conduziu a um interesse crescente no entendimento de suas implicações na saúde humana, no funcionamento dos ecossistemas, no tempo e, possivelmente, no clima, como um contribuinte para o aquecimento global (Imhoff et al., 2010).

Nos últimos anos têm sido observados aumentos significativos na temperatura de diferentes cidades do mundo (IPCC, 2007; Marengo, 2001; Kalnay e Cai, 2003). Sobre a bacia Amazônica, Marengo (2001) estimou o aquecimento na ordem de $+0.85^{\circ} \mathrm{C} / 100$ anos. Esse autor mostrou ainda que, o aquecimento pode variar por região, e pode se dar por causa de fatores naturais ou fatores antropogênicos como ilha de calor e o efeito de urbanização das grandes cidades, ou devido à combinação dos dois. Mudanças na superfície terrestre, como a urbanização, que tem como efeito a substituição de superfícies naturais por edificações, têm aumentado a impermeabilização dos solos e a irradiação de calor para a atmosfera (Sousa e Ferreira, 2012; Chen et al., 2006). Uma das mais conhecidas influências antropogênicas no clima é o fenômeno de aquecimento urbano. O aumento da temperatura da superfície ou do ar sobre uma área urbana em relação às áreas rurais ou suburbanas vizinhas é denominado Ilha de Calor (IC) (Arya, 2001; Freitas e Dias, 2005). E a diferença da temperatura do ar de uma área urbana em relação à sua vizinhança fornece a intensidade da IC (Memon et al., 2009; Hung et al., 2006).

Sabe-se que a IC urbana não resulta da ação de um simples fator, e sim das características da superfície, bem como da atmosfera urbana, dentre as quais se cita: a alta capacidade calorífica dos materiais de construção; a redução de áreas evapotranspirantes; a retenção de radiação de ondas longas devido ao aumento da poluição atmosférica (Arya, 2001; Gartland, 2010).

Pioneiramente, no Brasil, o fenômeno de IC foi estudado utilizando sensoriamento remoto por Lombardo (1985), que o analisou na metrópole de São Paulo utilizando os dados do satélite NOAA e encontrou diferenças de até $10{ }^{\circ} \mathrm{C}$, sendo que os maiores gradientes térmicos foram encontrados no horário de 15 e $21 \mathrm{~h}$ (hora local). Atualmente, existe uma significativa quantidade e disponibilidade de dados de sensores obtidos a partir de plataformas orbitais para o estudo de IC (Streutker, 2002). Tomlinson et al. (2011) listaram alguns sensores em operação, os quais disponibilizam os dados na faixa do infravermelho termal: Landsat ETM; MODIS/Aqua e MODIS/Terra; ASTER/Terra; AVHRR; AATSR e SEVIRI. Dentre estes, os dados do sensor MODIS (Moderate Resolution Imaging Spectroradiometer) têm sido amplamente utiliza- dos devido à sua alta resolução espacial e temporal (Freitas et al., 2011).

Hung et al. (2006) analisaram, com o uso do sensor MODIS/Terra, a intensidade (diária e sazonal) e o padrão espacial do fenômeno de IC para oito cidades da Ásia, utilizando temperatura de superfície e índice de vegetação: Tóquio, Beijing, Shanghai, Seoul, e Pyongyang (localizadas na zona climática temperada) e Bangkok, Manila e Ho Chi Minh (na zona tropical), no período de 2000-2003. Dentre as cidades de clima temperado, a maior intensidade da IC foi encontrada em Tóquio $\left(12{ }^{\circ} \mathrm{C}\right)$, e a menor, em Pyongyang $\left(4^{\circ} \mathrm{C}\right)$, para ambas em agosto de 2001. E para as cidades tropicais foram observadas diferenças de até $8{ }^{\circ} \mathrm{C}$ para Bangkok (em fevereiro de 2002) e de $7{ }^{\circ} \mathrm{C}$ para a cidade de Manila (em novembro de 2001).

Imhoff et al. (2010), em um estudo para três anos (2003-2005) do fenômeno de IC em 38 cidades mais populosas dos Estados Unidos, utilizando os satélites Landsat (sensor ETM+) e IKONOS estimaram um Índice de Áreas Impermeável (ISA) que foi comparado com a TS e o índice de vegetação, ambos estimados pelo sensor MODIS/Aqua. Eles compararam as intensidades da IC para diferentes biomas e encontraram valores de $6,3{ }^{\circ} \mathrm{C}$ a $9,0{ }^{\circ} \mathrm{C}$, para o período de verão naquelas cidades.

Peng et al. (2012) utilizaram o sensor MODIS/Aqua para analisar a TS e estimar a intensidade da IC em 419 cidades do globo, das quais 65 encontravam-se na América do Sul. Nas cidades da América do Sul as intensidades diurnas da IC foram de $3,0 \pm 1,4{ }^{\circ} \mathrm{C}$. Os autores mostraram também que a vegetação tem o papel de diminuir a intensidade da IC. Sousa e Ferreira (2012) utilizaram o sensor MODIS/Terra juntamente com os sensores TM e ETM+ dos Landsats 5 e 7 para estudar o fenômeno IC no município de Goiânia/GO, para o período seco nos anos de 2002 a 2011 e encontraram TS de até $38^{\circ} \mathrm{C}$, e intensidade da IC de $8{ }^{\circ} \mathrm{C}$.

Diversas metodologias têm sido utilizadas para o estudo de IC (Stewart, 2011). E apesar dos avanços no aperfeiçoamento de métodos para estudo de IC, ainda são poucos os trabalhos que utilizam conjuntamente as técnicas de sensoriamento remoto e a modelagem da superfície e da atmosfera de áreas urbanas para este fim. Nesse sentido, ressalta-se que é essencial a inclusão de dados de satélites em modelos climáticos regionais e globais (Jin e Shepherd, 2005). Além disso, vários modelos meteorológicos já dispõem de parametrizações físicas retratando as áreas urbanas com efeito direto nas estimativas do balanço de radiação, energia e água à superfície e, portanto, capazes de representar os efeitos dessas estruturas no clima local (Chen et al., 2011; Chen e Dudhia, 2001a; 2001b). Dentre esses modelos, cita-se o Modelo de Pesquisa e Previsão de Tempo (Weather Research and Forecasting-WRF) desenvolvido pelo Centro Nacional de Pesquisas Atmosféricas (National Center for Atmospheric Research-NCAR) em parceria com outros grupos de pesquisa. Os avanços da 
capacidade dos modelos para simular áreas urbanas com resolução de $1 \mathrm{~km}$ ou menos contribuíram para o aprimoramento dos estudos de IC, juntamente com avanços consideráveis nas especificações técnicas, principalmente em se tratando das resoluções espacial, espectral e temporal dos dados de sensoriamento remoto.

O modelo WRF já vem sendo usado para o estudo de IC em áreas metropolitanas como Beijing, Guangzhou, Hong Kong, Houston, New York, Salt Lake City, Tapei e Tóquio (Miao et al., 2009a; Chen et al., 2011). No entanto, o mesmo ainda não foi aplicado ao estudo de IC na América do Sul. Especificamente em Manaus, cujo índice de urbanização vem crescendo desde a década de 60 até os dias atuais (Silva, 2009), fomentado pelo aumento da população urbana, que entre 2000 e 2010 , passou de 1.405 .835 para 1.802.014 habitantes (IBGE, 2010), Maitelli e Wright (1996), com o uso de estações fixas e localizadas em três áreas distintas, sendo uma na Reserva Ducke (floresta), e outras na Fazenda Dimona (pastagem) e na área urbana da cidade, analisaram o efeito da IC para os meses chuvosos e secos de 1991 a 1992. Eles encontraram intensidade da IC média de $1,37^{\circ} \mathrm{C}$, comparado com a área de pastagem, e de $0,96{ }^{\circ} \mathrm{C}$ em relação à floresta para todo o período de estudo. $\mathrm{E}$, para o mês de setembro, a maior intensidade da IC encontrada foi de $2,5^{\circ} \mathrm{C}$ ocorrida às 11 horas da manhã.

Silva (2009), analisando a temperatura em 13 pontos da cidade de Manaus, observaram variações térmicas espaciais de $8,0^{\circ} \mathrm{C}$ e $6,0^{\circ} \mathrm{C}$ em agosto de 2008 e março de 2009 , respectivamente, sendo a zona centro-sul a que apresentou maior temperatura e a zona leste apresentou menor temperatura. Em um estudo mais recente, Souza e Alvalá (2014) analisaram a influência do fenômeno de IC no microclima da cidade de Manaus utilizando os dados de estações meteorológicas e resultados de simulações com o modelo Brazilian Regional Atmospheric Modeling System (BRAMS), considerando o período de 2000 a 2008 , e encontraram intensidade da IC média de $2,27{ }^{\circ} \mathrm{C}$ para a estação seca, com dois picos: um às 8 horas e outro às 17 horas.

A circulação de escala local é interligada com os processos do balanço de energia e radiação a superfície que são determinantes da temperatura da superfície. O sensoriamento remoto representa hoje uma fonte essencial de dados para o estudo dos processos de superfície e validação dos modelos meteorológicos e, portanto, para previsão nas meso e micro escalas (Chen et al., 2011). Este estudo utiliza o modelo meteorológico WRF em alta resolução espacial, como uma ferramenta de ligação, para analisar as simulações da temperatura da superfície e do ar a $2 \mathrm{~m}$, a partir de dados do sensor MODIS e as observações meteorológicas próximas à superfície, com o objetivo de demonstrar a capacidade do modelo para representar a distribuição espacial e os efeitos na baixa atmosfera do fenômeno da ilha de calor urbana na cidade de Manaus.

Como o modelo WRF não foi ainda usado para estudar o fenômeno de IC na América do Sul, o presente trabalho visa avaliar o uso deste modelo para estudar o fenômeno de IC em Manaus. Inicialmente a abrangência espacial do efeito de ilha de calor urbanana temperatura da superfície é avaliada para o período de 2002 a 2012 através do dados MODIS. Em seguida, o modelo WRF é usado para simular os campos de TS e TA de agosto e setembro de 2009 na área de Manaus. O desempenho do modelo para simular TS é avaliado usando-se TS do satélite, e para simular TA, serão feitas comparações com as observações. Finalmente, as intensidades da IC em Manaus estimadas com os dados de TA simulados e observados serão comparadas.

\section{Materiais e Métodos}

A área de estudo (Fig. 1) compreende a cidade de Manaus/AM localizada nas coordenadas $3^{\circ} \mathrm{S}$ e $60^{\circ} \mathrm{W}$, à esquerda dos rios Negro e Amazonas. Os dados utilizados são os campos de TS dos meses de agosto e setembro dos quatro primeiros (2002-2005) e dos quatro últimos anos (2009-2012) da série de dados remotos do satélite ambiental Aqua, com resolução de $1 \mathrm{~km}$ por $1 \mathrm{~km}$, produto MYD11A2, obtidos no portal do sensor MODIS na NASA (National Aeronautics and Space Administration), através do LP DAAC (Land Processes Distributed Active Archive Center), disponível em: https://lpdaac.usgs.gov/get_data/data_pool e que possuem alta confiabilidade (Wan, 2007; 2008). Em vista de que os dados de TS do sensor MODIS/Aqua já foram validados

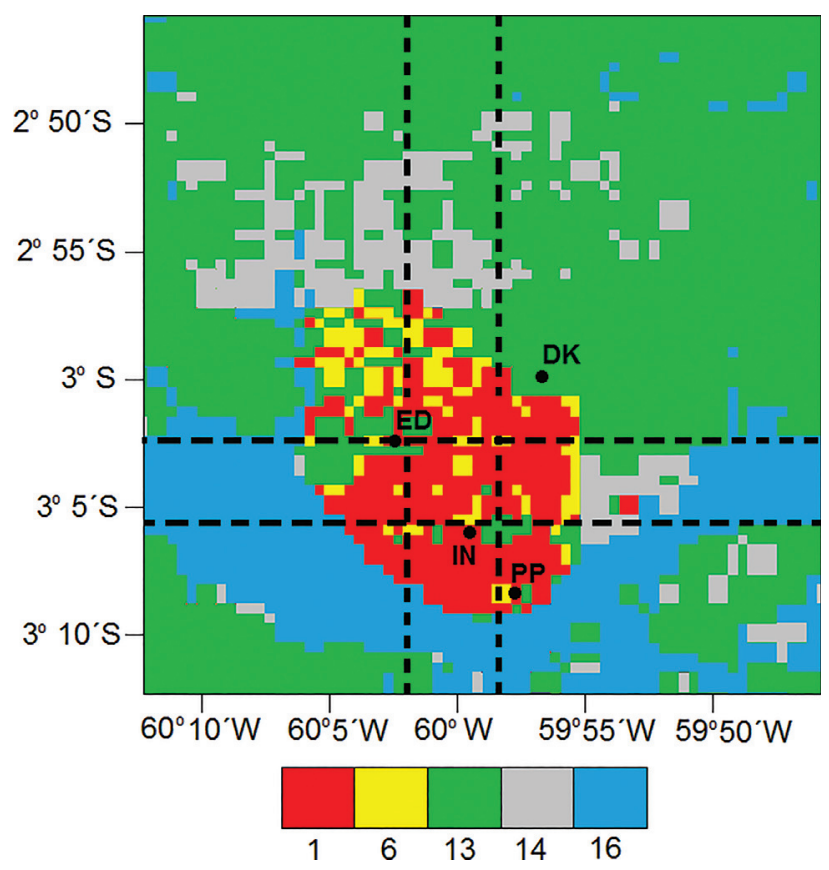

Figura 1 - Região geográfica da cidade de Manaus, incluindo as classes de cobertura da superfície do modelo WRF (1: área urbana; 6: mosaico de vegetação; 13: floresta ombrófila densa; 14: mosaico de pastagem/agricultura; 16: água), juntamente com a localização das estações meteorológicas (ED: aeroporto Eduardo Gomes; DK: Reserva Ducke; IN: INPA; PP: aeroporto Ponta Pelada). 
(Wan, 2008), estes foram inicialmente usados para obter os campos de TS dos períodos de 2002-2005 e de 2009-2012. Ressalta-se que a análise separada dos campos de TS do satélite para dois períodos (de 2002 a 2005 e de 2009 a 2012) deve-se ao interesse em inferir a dinâmica de urbanização da cidade de Manaus, que foi mais lenta no primeiro período (2002 a 2005) e mais acentuada no segundo período (2009 a 2012). Para fins de comparação o mesmo número de anos foi usado para os dois períodos. Os campos de TS são representativos do horário de passagem do satélite (13 h 30 min-hora local).

As condições iniciais e de contorno da atmosfera para as simulações com o modelo WRF foram os dados de reanálise CFSR (Climate Forecast System Reanalysis), com resolução de $50 \mathrm{~km}$ por $50 \mathrm{~km}$ obtidos no site: http://soostrc.comet.ucar.edu/data/grib/cfsr/2009/, e de temperatura da superfície do mar disponíveis em: ftp://ftp.emc.ncep.noaa.gov/cmb/sst/oisst_v2/GRIB obtidos do NCEP (National Centers for Environmental Prediction). O mapa de uso e cobertura do solo da área urbana de Manaus foi definido com base em Roque (2006), e é mostrado na Fig. 1.

Os dados de TA foram obtidos das estações meteorológicas dos aeroportos Eduardo Gomes $\left(3^{\circ} 02^{\prime} 28^{\prime \prime} \mathrm{S}\right.$; $60^{\circ} 03^{\prime} 02^{\prime \prime}$ O) e Ponta Pelada ( $3^{\circ} 8^{\prime} 45^{\prime \prime} \mathrm{S}$; 59 59'39” O) disponíveis no site, http://bancodedados.cptec.inpe.br/, do Instituto Nacional de Pesquisas Espaciais (INPE) e da estação do Instituto Nacional de Pesquisas da Amazônia (INPA) ( $3^{\circ} 05^{\prime} 45^{\prime \prime}$ S; 59 59'12" O), e da Reserva Ducke (259'42” S; 59॰56’6" O). As localizações destas estações e áreas estão indicadas na Fig. 1.

Em vista de que agosto e setembro são os meses mais quentes e com menor nebulosidade em Manaus (Fisch et al., 1998), e que o interesse do presente artigo é sobre IC nesta cidade, as análises foram feitas apenas para estes meses.

Para o estudo de caso, as análises foram para agosto e setembro de 2009, por esta estação ter sido anomalamente seca em relação à média climatológica do período de $1960 \mathrm{a}$ 1990. Neste estudo, foram usados os dados de TS do satélite e os resultados das simulações com o WRF para comparar a variação espacial desta variável. Além disso, os ciclos diários da TA observada nas estações meteorológicas e simulada pelo modelo WRF, nas respectivas coordenadas das estações meteorológicas, foram analisados e comparados. Estas análises também foram para os meses de agosto e setembro de 2009.

O WRF versão 3.1 .1 foi configurado com as parametrizações físicas de radiação solar, de ondas longas, convecção e esquema de superfície, conforme descrito em Skamarock et al. (2005). O WRF foi configurado em dois domínios abrangendo a região metropolitana de Manaus, sendo o primeiro com resolução espacial de $5 \mathrm{~km}$ por $5 \mathrm{~km}$, e o segundo de $1 \mathrm{~km}$ por $1 \mathrm{~km}$, este último aninhado ao primeiro. A atmosfera foi discretizada em 38 níveis verti- cais em coordenadas sigma-eta (híbrida) desde a superfície até $50 \mathrm{hPa}$. As análises aqui apresentadas referem-se apenas ao segundo domínio.

A simulação foi inicializada às 12 UTC do dia 25 de julho de 2009 e conduzida, continuamente, até o dia $1^{\circ}$ de outubro do mesmo ano. As opções físicas utilizadas no modelo foram: parametrização de cúmulos (5, 5 -Grell Deveni ensemble scheme); microfísica (2, 2 -Lin et al. scheme); radiação de onda longa (4, $4-R R T M G$ scheme); radiação de onda curta (4, 4 - RRTMG scheme); física da superfície (2, 2 -Noah Land Surface Model), física urbana (1, 1 -Urban canopy model); camada limite planetária $(1,1$ -YSU scheme). Também, usou-se o mapa de cobertura de superfície da área urbana, definido por Roque (2006), para modificar os ambientes definidos inicialmente pelo mapa de cobertura da superfície do USGS referentes às áreas de floresta, rios e área urbana.

O desempenho do modelo para simular TS foi avaliado comparando-se os campos de TS obtidos com os dados de satélite e do modelo, bem como comparando as variações da TS do modelo e do satélite ao longo de uma seção média longitudinal e uma seção média latitudinal, que foram selecionadas de forma que estas cruzassem diferentes superfícies (floresta, área urbana e rios). As seções médias longitudinal e latitudinal foram de $-60,03^{\circ}$ a $-59,97^{\circ} \mathrm{e}-3,09^{\circ}$ a $-3,03^{\circ}$, respectivamente. As localizações destas duas seções estão indicadas pelas retas tracejadas na Fig. 1.

Para validar as simulações de TA, foram utilizadas as medidas experimentais de TA em estações meteorológicas localizadas na área urbana (aeroportos e INPA) e em área de floresta (Reserva Ducke), cujas localizações são ilustradas na Fig. 1.

\section{Resultados e Discussões}

\subsection{Análise de TS para os períodos de 2002-2005 e 2009-2012: dados MODIS}

As Figs. 2 e 3 apresentam os campos espaciais de TS em Manaus média de agosto e setembro para os períodos de 2002-2005 e 2009-2012, respectivamente, derivados do sensor MODIS. Nos dois períodos, os maiores valores de TS ocorreram em setembro, e se localizaram sobre duas regiões da cidade: uma envolvendo a maior parte da zona Sul e outra na intersecção das zonas Leste e Norte da cidade de Manaus. Nas regiões Centro-Sul e Centro-Oeste da cidade observaram-se valores de TS entre $38^{\circ} \mathrm{C}$ e $40^{\circ} \mathrm{C}$, e nas demais regiões, valores entre $30^{\circ} \mathrm{C}$ e $38^{\circ} \mathrm{C}$, sendo que os valores inferiores a estes correspondem a regiões de rios e/ou florestas. Este resultado coincide exatamente com a localização das manchas urbanas que apresentam maior densidade populacional conforme dados do Censo 2010 (IBGE, 2012). Por outro lado, na porção oeste da cidade, onde ainda existem áreas verdes preservadas englobando as proximidades do Aeroporto Internacional Eduardo Gomes, 

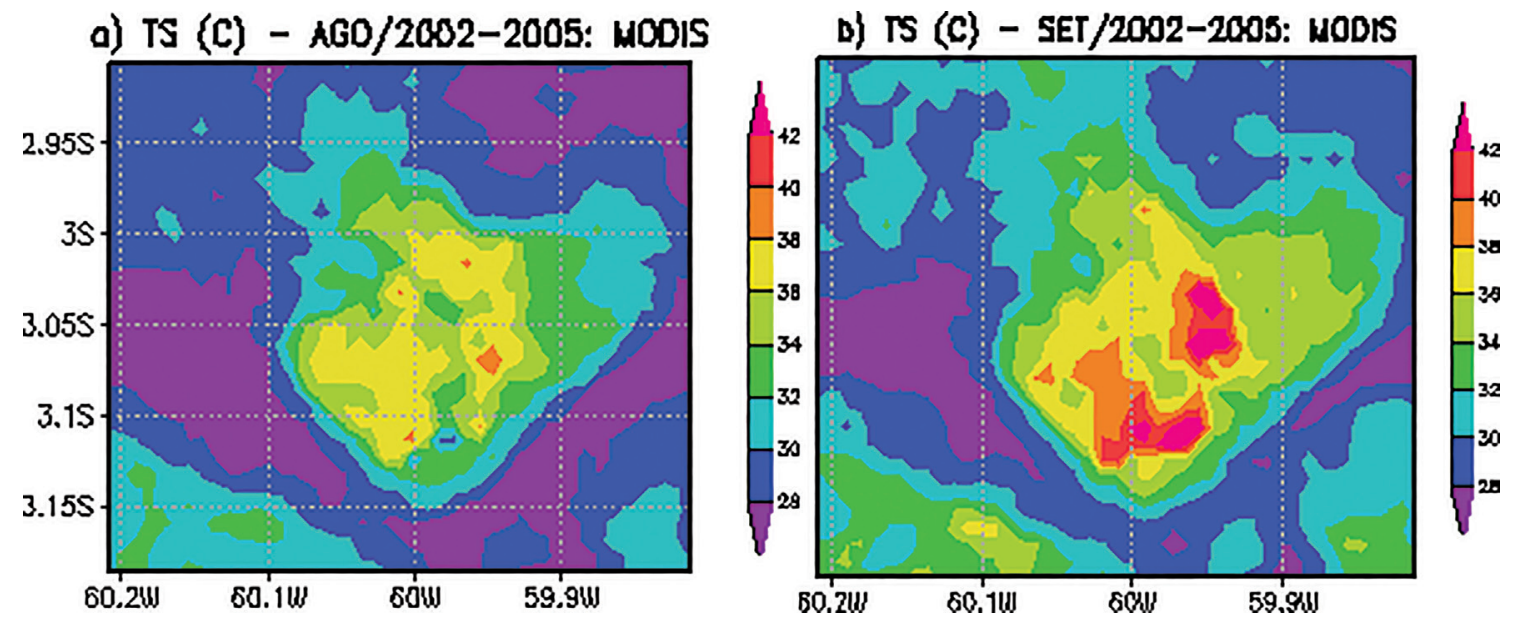

Figura 2 - Temperatura média da superfície $\left({ }^{\circ} \mathrm{C}\right)$ estimada pelo MODIS para os meses de: (a) agosto e (b) setembro para o período de 2002 a 2005 , sobre a cidade de Manaus, com resolução espacial de $1 \mathrm{~km}$ por $1 \mathrm{~km}$.

a) TS (C) - AGO/2009-2012: MODIS

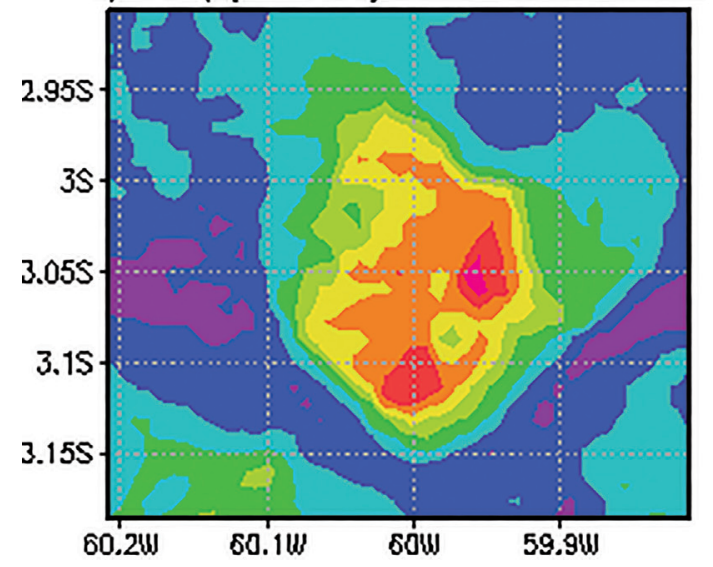

D) TS (C) - SET/2009-2012: HODIS

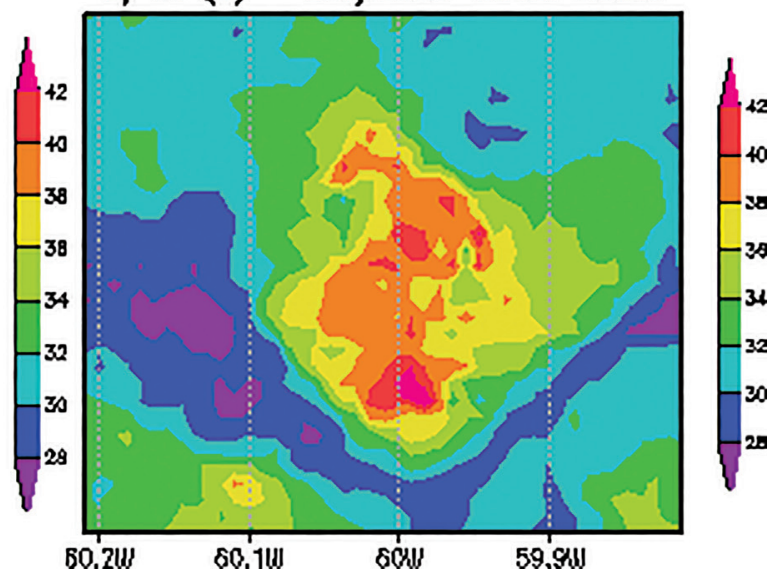

Figura 3 - Temperatura média da superfície $\left({ }^{\circ} \mathrm{C}\right)$ estimada pelo MODIS para os meses de: (a) agosto e (b) setembro para o período de 2009 a 2012 , sobre a cidade de Manaus, com resolução espacial de $1 \mathrm{~km}$ por $1 \mathrm{~km}$.

a TS foi mais amena. Da mesma forma, na região que envolve a mata do campus da Universidade Federal do Amazonas (UFAM) e nas porções no entorno da cidade, observaram-se TS amenas. No caso do contorno sul, a TS sofre influência dos rios e, nas porções nos contornos leste e oeste, além da influencia dos rios tem-se a presença de áreas de floresta.

É possível observar que nos quatro últimos anos do período analisado em relação aos quatro primeiros anos, houve um aumento na TS média em diferentes bairros de Manaus, nos dois meses, e em particular em setembro. Algumas áreas em que a TS era menor nos primeiros anos passaram a apresentar TS maiores nos últimos anos, possivelmente pelo fato de que áreas onde não havia construções (casas, prédios, ruas) passaram a ser mais densamente povoadas ou ainda porque as construções avançaram sobre as áreas verdes (dentro da cidade) ou de florestas nas adjacências da cidade, conforme aponta Souza e Alvalá (2014). Somado a isso, ressalta-se a substituição de materiais de baixa capacidade térmica por materiais de alta capacidade térmica utilizados na construção civil e pavimentos das ruas.

\subsection{Análise de TS ago-set-2009: WRF x MODIS}

A Fig. 4 mostra a TS média dos valores de agosto e setembro de 2009, obtidos pelo sensor MODIS. Nota-se que a distribuição espacial da TS seguiu o mesmo padrão que o dos campos de TS médias dos períodos de 2002-2005 e 2009-2012, bem como em relação aos valores que foram próximos aos destes campos. Evidencia-se nesta figura o intervalo de variação espacial com as maiores TS, entre $38^{\circ} \mathrm{C} \mathrm{e} 42^{\circ} \mathrm{C}$, e as menores em torno de $30^{\circ} \mathrm{C}$, o que resulta numa diferença de $12{ }^{\circ} \mathrm{C}$. As áreas de TS menores que $30^{\circ} \mathrm{C}$ estão ao sul, sudoeste e sudeste das regiões de rios e florestas.

O campo de TS média dos valores diários de 01 de agosto a 30 de setembro de 2009 gerados pelo modelo WRF (Fig. 5) mostra padrão similar ao correspondente campo de 


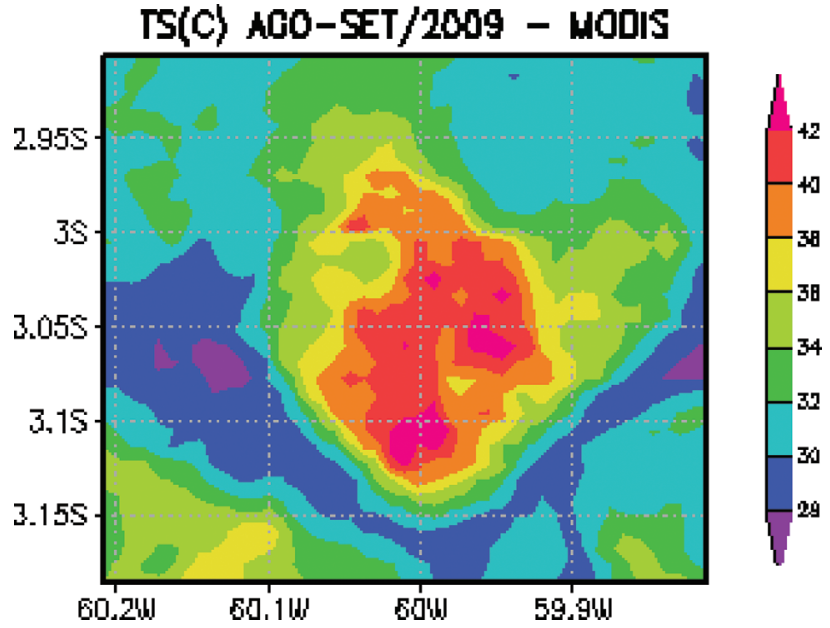

Figura 4 - Temperatura da superfície $\left({ }^{\circ} \mathrm{C}\right)$ média para os meses de agosto e setembro de 2009, sobre a cidade de Manaus na resolução de $1 \mathrm{~km}$ por $1 \mathrm{~km}$, estimada pelo MODIS.

TS construído com os dados de satélite. Entretanto, o campo simulado tem maior homogeneidade, uma vez que a definição da cobertura da superfície foi limitada a três classes (florestas, rios e área urbana). No campo de TS simulado, são encontrados valores entre $40{ }^{\circ} \mathrm{C}$ e $42{ }^{\circ} \mathrm{C}$ para a maior parte da área urbana, envolvendo as zonas Sul, Centro-Sul e Centro-Oeste da cidade, entre $36^{\circ} \mathrm{C}$ e $38^{\circ} \mathrm{C}$ para a área de floresta, e cerca de $30^{\circ} \mathrm{C}$ para a superfície de rios.

Analisando a diferença entre os dados do modelo e do satélite (Fig. 6), observa-se que o modelo superestimou a TS em até $6{ }^{\circ} \mathrm{C}$ em determinadas áreas de floresta (área da Reserva Ducke) e subestimou em até $6{ }^{\circ} \mathrm{C}$ em determinada porção da superfície do rio. Entretanto, para as regiões que apresentaram TS mais elevadas (maior parte da área urba-

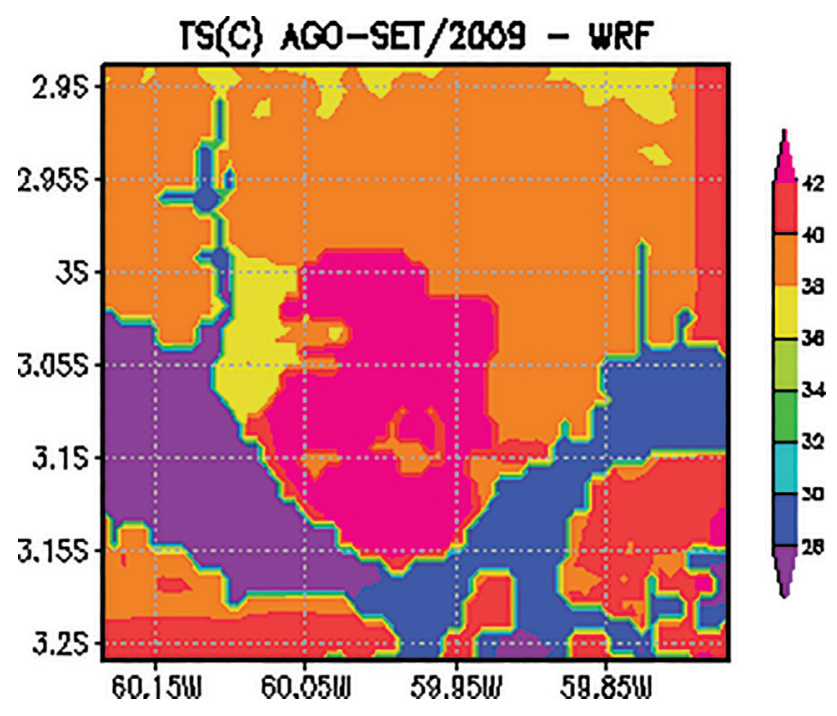

Figura 5 - Temperatura da superfície $\left({ }^{\circ} \mathrm{C}\right)$ para o período de 01 de agosto a 30 de setembro de 2009 na resolução $1 \mathrm{~km}$ por $1 \mathrm{~km}$, simulada com o modelo WRF.

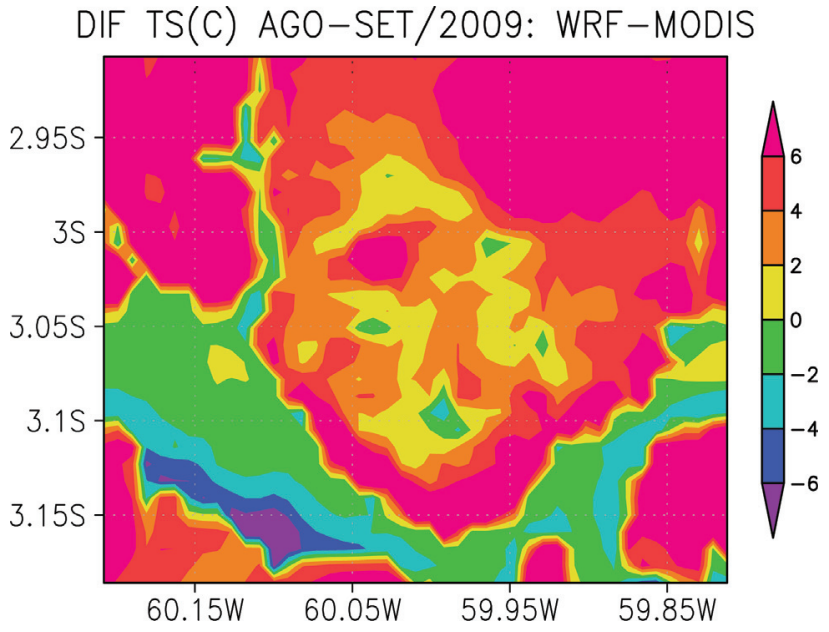

Figura 6 - Diferença entre a temperatura da superfície $\left({ }^{\circ} \mathrm{C}\right)$ simulada pelo modelo WRF e a estimada pelo MODIS.

na), a diferença entre modelo e satélite é de cerca de $+2{ }^{\circ} \mathrm{C}$ ou $-2{ }^{\circ} \mathrm{C}$. Assim, conclui-se que o modelo apresentou-se mais eficiente para representar o padrão espacial de TS na área urbana.

Ao longo da seção longitudinal (Fig. 7), os dados do modelo WRF mostraram valores de $\mathrm{TS}$ de $30{ }^{\circ} \mathrm{C}$ na área de floresta, $43{ }^{\circ} \mathrm{C}$ na área urbana e $28^{\circ} \mathrm{C}$ na área de rio, o que implica em diferenças de TS entre a área urbana e demais áreas de até $13^{\circ} \mathrm{C}$, para a área de floresta, e de até $15^{\circ} \mathrm{C}$, para a área de rio. Quanto aos dados do sensor MODIS, os valores de TS nas áreas de floresta e de rios são os mesmos dos simulados nestas áreas, e de $42{ }^{\circ} \mathrm{C}$ para área urbana, o que resulta em diferenças de TS entre área urbana e demais áreas de $12{ }^{\circ} \mathrm{C}$ para área de floresta e de $14{ }^{\circ} \mathrm{C}$ para área de rio. Ressalta-se que nestas estimativas de diferenças de temperatura foram utilizados os valores médios máximos e médios mínimos para cada ambiente (floresta, rio e área urbana).

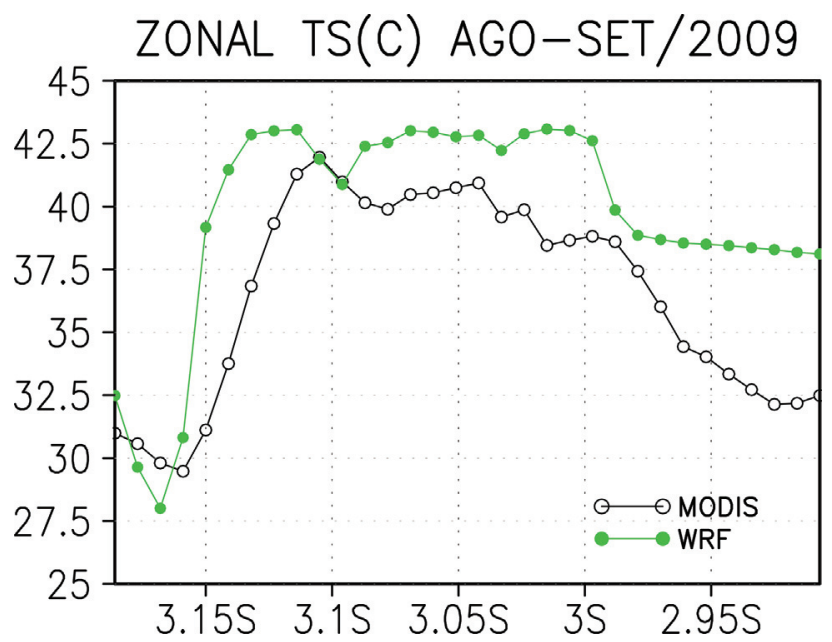

Figura 7 - Seção média longitudinal das temperaturas da superfície $\left({ }^{\circ} \mathrm{C}\right)$ estimadas pelo MODIS e simuladas pelo modelo WRF. 
Na seção latitudinal média (Fig. 8), os valores de TS simulados pelo modelo WRF variaram de $38^{\circ} \mathrm{C}$ na área de floresta, $43{ }^{\circ} \mathrm{C}$ na área urbana, e $28{ }^{\circ} \mathrm{C}$ na área de rios, resultando em diferença de $5{ }^{\circ} \mathrm{C}$ em relação à área de floresta e de $15^{\circ} \mathrm{C}$ em relação à área de rios. Os valores de TS derivados das imagens de satélite foram de $31^{\circ} \mathrm{C}$, para a área de floresta, $41^{\circ} \mathrm{C}$ para a área urbana, e cerca de $29^{\circ} \mathrm{C}$ para área de rios. Em consequência as diferenças foram de $10^{\circ} \mathrm{C}$ em relação à floresta e de $12^{\circ} \mathrm{C}$, em relação aos rios.

As diferenças entre o máximo e o mínimo das curvas da simulação (WRF) e do satélite (SAT) refletem o efeito da variação da densidade de urbanização na TS e a similaridade entre estas curvas indica que o modelo WRF apresenta consistência com as medidas remotas. No entanto, a curva do gráfico que representa os dados por satélite é mais suave em relação ao modelo, pois a simulação (WRF) considera apenas três classes de cobertura do solo, enquanto o satélite considera maior diversidade dessas coberturas.

\subsection{Análise de TA ago-set-2009: WRF x Observações}

Com o intuito de validar os valores de TA simulados pelo modelo WRF, foram analisados os ciclos diários das TA medidas nas estações meteorológicas localizadas em: 1) área de floresta na Reserva Ducke (DUCKE); e 2) áreas urbanas - INPA, Aeroportos de Ponta Pelada (P. PELADA) e Eduardo Gomes (E.GOMES), e as simuladas pelo modelo WRF para os ambientes de floresta (DUCKE-WRF) e urbano (INPA-WRF; P.PELADA-WRF e E.GOMES-WRF) nas mesmas coordenadas geográficas das estações meteorológicas, para o período de agosto a setembro de 2009.

Nos dados do modelo WRF (triangulo cheio), a TA máxima ocorreu com um atraso de uma hora em relação a das estações, exceto na estação EDUARDO GOMES. Embora o modelo tenha representado adequadamente as variações horárias do ciclo diário, subestimou a TA no turno da manhã (entre 6 horas e 12 horas) e superestimou-a no restante do dia. Este fato está associado, possivelmente, às

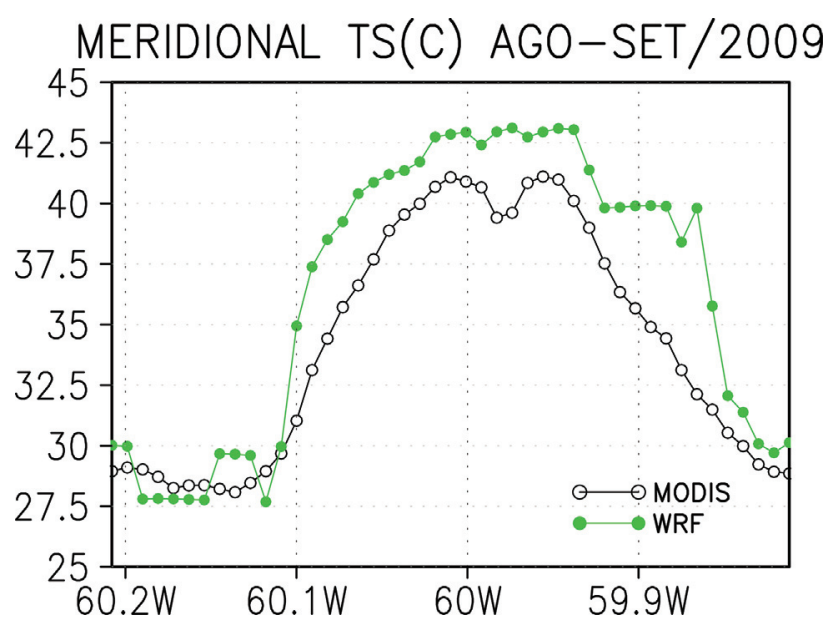

Figura 8 - Seção média latitudinal das temperaturas da superfície $\left({ }^{\circ} \mathrm{C}\right)$ estimadas pelo MODIS e simuladas pelo modelo WRF. diferenças na composição da capacidade térmica do modelo em comparação aos dados medidos. No modelo um único valor é atribuído para ser representativo de uma grade de $1 \mathrm{~km} \times 1 \mathrm{~km}$, enquanto nos locais de medidas a composição do mosaico urbano é complexa, envolvendo elementos de concreto, asfalto de vegetação que contribuem para definir TS e TA localmente. Observa-se ainda que o modelo superestimou as TA máximas (horário de pico - entre 12 horas e 15 horas) em PONTA PELADA e EDUARDO GOMES com valores de $34,21{ }^{\circ} \mathrm{C}$ e $34,29{ }^{\circ} \mathrm{C}$, em relação aos medidos de $33,26{ }^{\circ} \mathrm{C}$ e $32,68{ }^{\circ} \mathrm{C}$, respectivamente (Figs. 9a e 9b). No entanto, o modelo apresentou valores menores $\left(33,47{ }^{\circ} \mathrm{C}\right.$ e $\left.33,57{ }^{\circ} \mathrm{C}\right)$, respectivamente, para as estações do INPA $\left(33,98{ }^{\circ} \mathrm{C}\right)$ e R. DUCKE $\left(33,67^{\circ} \mathrm{C}\right)$ (Figs. 9a e 9c).

A magnitude da intensidade da IC e seu ciclo diário são avaliados comparando-se as diferenças de TA entre áreas urbanas e de floresta, esta última representando a paisagem típica do entorno norte/leste de Manaus, para as simulações e observações. Medidas de TA sobre as áreas de rio não são disponíveis. Esses resultados são mostrados na Fig. 10.

A Fig. 10a apresenta os ciclos diários observados e simulados de TA para a área urbana médios das estações. A Fig. 10b mostra o ciclo diário das diferenças entre a TA média da área urbana e a TA observada da reserva Ducke (ESTACÃO (OBS)-DUCKE (OBS)). A Fig. 10c mostra o ciclo diário das diferenças entre a TA simulada média da área urbana e a TA observada da reserva Ducke (WRFDUCKE). No caso ESTAÇÃO (OBS) - DUCKE (OBS), os gráficos mostram dois picos na intensidade da IC: um às 6 horas $\left(3,90^{\circ} \mathrm{C}\right)$ e outro às 18 horas $\left(2,8^{\circ} \mathrm{C}\right)$, o que está de acordo com Souza (2012). E no caso WRF-DUCKE, ocorreu um pico às 18 horas $\left(4,95^{\circ} \mathrm{C}\right)$, e o pico no horário de 6 horas não foi reproduzido. Isto certamente porque as simulações superestimaram os valores de TA no turno matutino. Entretanto, nota-se que há coerência entre os ciclos diários das intensidades da IC dos dados medidos e dos dados simulados, em particular após às 12 horas. Na média os valores de intensidade de IC para o caso ESTACÃO (OBS)-DUCKE(OBS), e WRF-DUCKE foram de $1,87^{\circ} \mathrm{C}$ e $1,80{ }^{\circ} \mathrm{C}$, respectivamente, o que mostra consistência. Portanto, os dados simulados de TA com o modelo WRF podem ser usados para fins de estimar a intensidade da IC em Manaus.

\section{Conclusões}

Com o uso de dados do sensor MODIS e aplicação do modelo WRF em altíssima resolução espacial foi possível identificar e quantificar áreas que apresentaram os maiores e menores valores da TS, localizando-as espacialmente para os períodos de 2002-2005 e de 2009-2012 e no estudo de caso para agosto e setembro de 2009. Enquanto que com o uso dos dados observados das estações meteorológicas foi possível a validação da TA simulada pelo modelo WRF, 

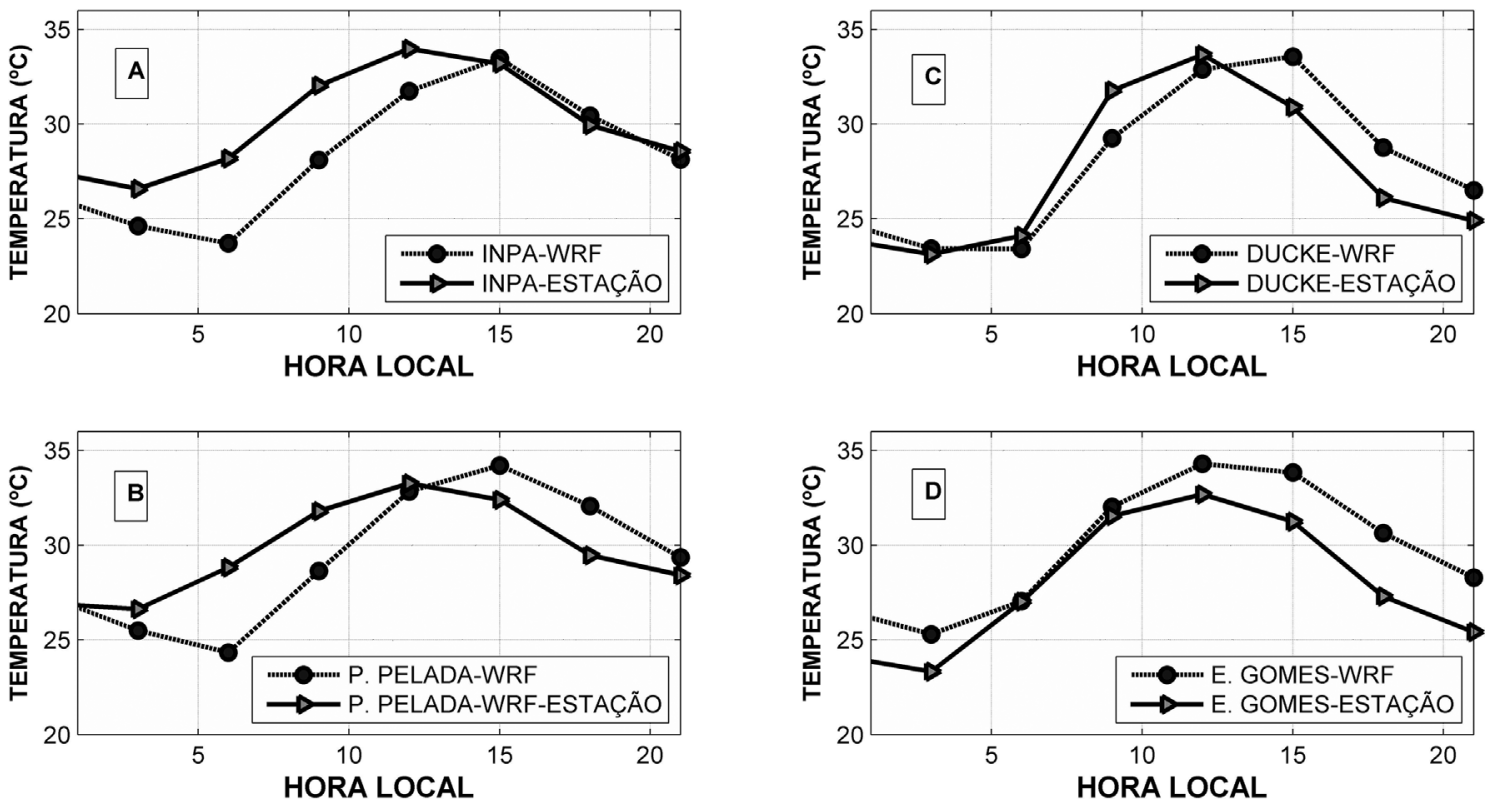

Figura 9 - Ciclo diário da temperatura do ar observada e simulada pelo modelo WRF: (a) estação INPA; (b) estação AEROPORTO PONTA PELADA, (c) estação RESERVA DUCKE, (d) estação AEROPORTO EDUARDO GOMES.
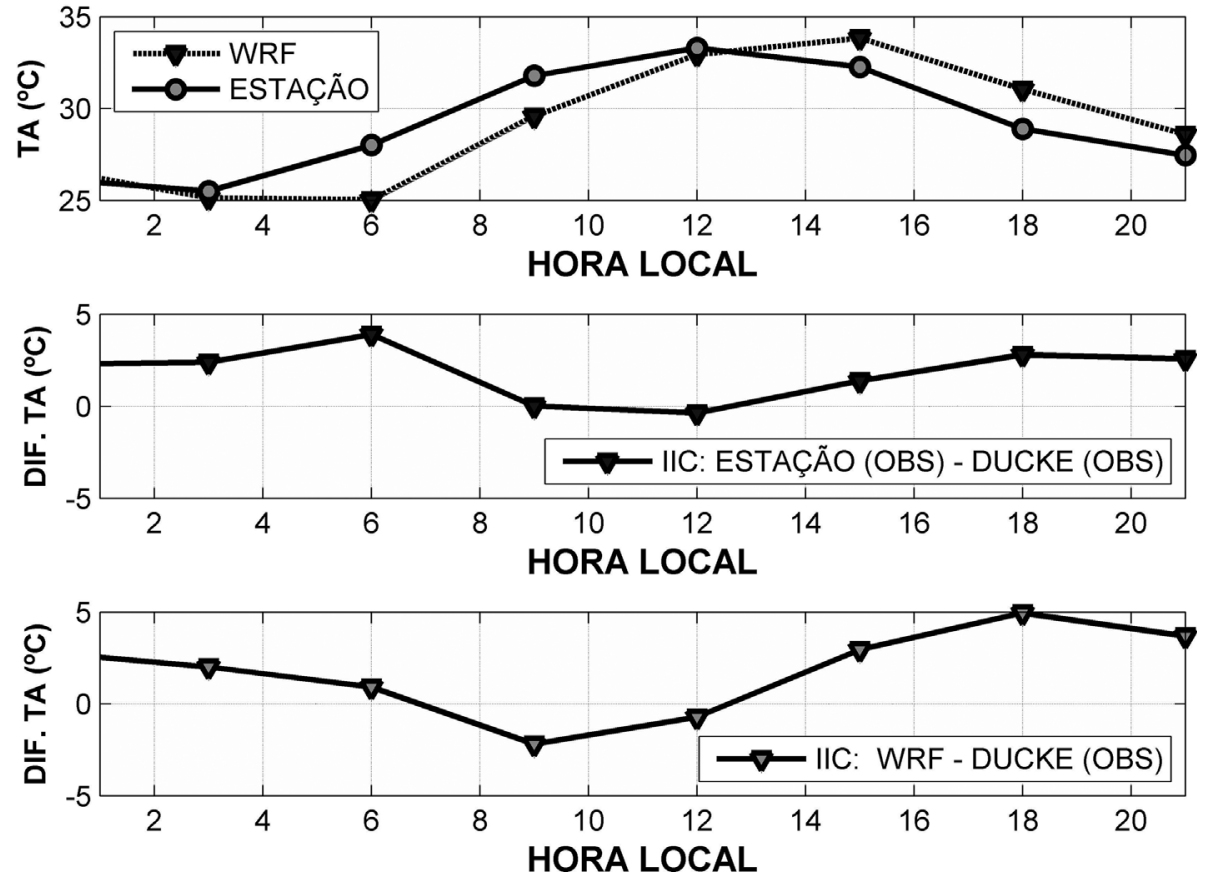

Figura 10 - (a) Ciclo diário das temperaturas do ar, para a área urbana, observado e simulado pelo WRF. (b) Diferença de temperatura do ar observada entre as estações urbanas e a estação na região de floresta. (c) Diferença entre a temperatura do ar na região urbana modelada pelo WRF e os dados observados nas estações.

assim como a estimativa da intensidade de IC observada e simulada em agosto e setembro de 2009.

Os campos de TS médios dos períodos de 2002-2005 e de 2009-2012 mostraram padrões espaciais similares, mas com valores maiores no segundo período. Isto decorre possivelmente pela urbanização mais acentuada no segundo período em relação ao primeiro. Os dados de TS do sen- sor MODIS e do modelo WRF apresentaram o mesmo padrão espacial. Esses resultados corroboram os estudos de Silva (2009) e Souza (2012).

Entretanto, a diferença de TS entre área urbana, de rios e floresta estimada pelos dados do modelo foi subestimada em determinadas regiões em relação à estimada com os dados de satélite. Isso decorreu do fato que o 
modelo apresentou maiores valores de TS para a área de floresta e menores valores para a área de rios, o que implica na necessidade de ajustes de parâmetros na física de superfície do modelo WRF. Na análise longitudinal, as diferenças com as TS do WRF e do satélite foram respectivamente de até $13^{\circ} \mathrm{Ce} 12^{\circ} \mathrm{C}$ em relação à floresta e de $15^{\circ} \mathrm{Ce} 14^{\circ} \mathrm{C}$ em relação aos rios. Portanto, a diferença destas entre o modelo e satélite foi de $1{ }^{\circ} \mathrm{C}$ tanto em relação à floresta como em relação aos rios. No entanto, na análise latitudinal, as diferenças com as TS do modelo e do satélite foram respectivamente de até $5{ }^{\circ} \mathrm{Ce} 10^{\circ} \mathrm{C}$ em relação à floresta, e de até $15{ }^{\circ} \mathrm{C}$ e $12{ }^{\circ} \mathrm{C}$, em relação aos rios. Neste caso, a diferença entre modelo e satélite foi maior para área de floresta do que para de rios. Isso se deve, possivelmente, porque as parametrizações da capacidade térmica no modelo WRF para estes ambientes precisam ser mais bem ajustadas, bem como as parametrizações na diversidade dos tipos de cobertura do solo.

Os dados de estações meteorológicas permitiram validar o ciclo diário da TA simulada pelo modelo WRF, bem como quantificar a intensidade da IC na cidade de Manaus. Os resultados mostraram que na média das estações, as variações horárias das TA observadas no ciclo diário foram reproduzidas pelo modelo, porém o modelo subestimou as TA entre 6 e 12 horas e superestimou no restante do dia.No entanto, para algumas estações e horários do dia os valores de TA observados e simulados são muito próximos. Isso ocorreu, por exemplo, com as TA máximas das estações do INPA e da R. DUCKE que apresentaram diferenças entre modelo e observação menores que $1{ }^{\circ} \mathrm{C}$. Por outro lado, a maior diferença da TA entre modelo e observação, foram notadas nas estações de aeroportos. Portanto, os resultados indicam que os dados simulados de TA podem ser úteis para monitorar a intensidade da IC considerando a área de floresta representativa do entorno da cidade. No entanto, as observações medidas em um número maior de estações deverão ser analisadas para resultados mais conclusivos e também refinar o campo de superfície do modelo considerando maior diversidade de classes de cobertura da superfície.

\section{Agradecimentos}

Os autores agradecem a FINEP (Projeto REMCLAM) e a FAPEAM (Projetos PROESTADO e GOAMAZON), pelo apoio financeiro à pesquisa. $\mathrm{O}$ primeiro autor agradece à Coordenação de Aperfeiçoamento de Pessoal (CAPES), pela concessão de bolsa de estudo. Os dois últimos autores foram parcialmente financiados pelo CNPq. Os autores agradecem as sugestões dos dois revisores anônimos.

\section{Referências}

ARYA, S.P. Introduction to Micrometereology. International Geophysics Series, v. 79. Academic Press, 2001.
CHEN, F.; KUSAKA, H.; BORNSTEIN R.; CHING J.; GRIMMOND, C.S.B; GROSSMAN-CLARKE S.; LORIDAN T.; MANNING K.W.; MARTILLI, A.; MIAO, S.; SAILOR, D.; SALAMANCA, F.P.; TAHA, H.; TEWARE, M.; WANG, X.; WYSZOGRODZKI, A.A.; ZHANG, C. The integrated WRF/urban modelling system: development, evaluation, and applications to urban environmental problems. International Journal of Climatology, v. 31, p. 273-288, 2011.

CHEN, F.; DUDHIA, J. Coupling an advanced land surface - hydrology model with the Penn State-NCAR MM5 modeling system. Part I: Model implementation and sensitivity. Monthly Weather Review,v. 129, p. 569-585, $2001 \mathrm{a}$.

CHEN, F.; DUDHIA, J. Coupling an advanced land surfacehydrology model with the Penn State-NCAR MM5 modeling system. Part II: Preliminary model validation. Monthly Weather Review,v. 129, p. 587-604, 2001 b.

CHEN; X.L.; ZHAO, H.M.; LI, P.X.; YIN, Z.Y. Remote sensing image-based analysis of the relationship between urban heat island and land use/cover changes. Remote Sensing of Environment, v. 104, p. 133-146, 2006.

FISCH, G.; MARENGO, J.A.; NOBRE, C.A. Uma revisão geral do clima da Amazônia. Acta Amazônica, v. 28, p. 101-126, 1998.

FREITAS, E.D.; DIAS, P.L.S. Alguns efeitos de áreas urbanas na geração de uma ilha de calor. Revista Brasileira de Meteorologia, v. 20, p. 355-366, 2005.

FREITAS, R.M.; ARAI, E.; ADAMI, M.; FERREIRA, A.S.; SATO, F.Y.; SHIMABUKURO, Y.E.; ROSA, R.R.; ANDERSON, L.O.; RUDORFF, B.F.T. Virtual laboratory of remote sensing time series: visualization of MODIS EVI2 data set over Sourth America. Journal of Computational Interdisciplinary Science, v. 2, p. 57-68, 2011.

GARTLAND, L. Ilhas de calor: como mitigar zonas de calor em áreas urbanas. Tradução: Silvia Helena Gonçalves. São Paulo: Oficina de Textos, 2010.

HUNG, T.; UCHIHAMA, D.; OCHI S.; YASUOKA, Y. Assessment with satellite data of the urban heat island effects in Asian mega cities. International Journal of Applied Earth Observation and Geoinformation, v. 8, p. 34-48, 2006.

JIN, M.; SHEPHERD, J.M. Inclusion of urban landscape in a climate model: How can satellite data help? Bulletin of the American Meteorological Society, v. 86, p. 681-689, 2005.

IBGE - Instituto Brasileiro de Geografia e Estatísica. (http://www.ibge.gov.br/home/estatistica/populacao/censo 2010/tabelas_pdf/Amazonas.pdf), Acesso em 31/12/2012.

IMHOFF, M.L.; ZHANG, P.; WOLFE, R.; BOUNOUA, L. Remote sensing of the urban heat island effect across biomes in the continental USA. Remote Sensing of Environment, v. 114, p. 504-513,2010.

IPCC. Summary for Policymakers, in Climate Change 2007: Impacts, Adaptation and Vulnerability. Contribution of Working Group II to the Fourth Assessment Report of the Intergovernmental Panel on Climate Change. edited by $\mathrm{M}$. L. Parry, O. F. Canziani, J. P. Palutikof, P. J. van der Linden and C. E. Hanson, pp. 7-22, Cambridge University Press, Cambridge, UK, 2007.

KALNAY, E.; CAI, M. Impact of urbanization and land-use change on climate. Nature, v. 423, p. 528-531, 2003. 
LOMBARDO, M.A. A ilha de calor nas metrópoles - O exemplo de São Paulo. São Paulo: Huciteck. 224 p, 1985.

LP-DAAC - Land Processes Distributed Active Archive Center. (https://lpdaac.usgs.gov). Acesso em 27/02/2012.

MAITELLI, G.T.; WRIGHT, I.R. The climate of a riverside city in the Amazon Basin: urban-rural differences in temperature and humidity. In: GASH, J.H.C.; NOBRE, C.A.; ROBERTS, J.M.; VICTORIA, R.L. (Eds.) Amazonian Deforestation and Climate, New York: J. M. Wiley e Sons, 1996, p. 193-206.

MARENGO, J.A. Mudanças climáticas globais e regionais: Avaliação do clima atual do Brasil e projeções de cenários climáticos do futuro. Revista Brasileira de Meteorologia, v. 16, p. 1-18, 2001.

MEMON, R.A.; LEUNG, D.Y.C.; LIU, C.H. An investigation of urban heat island intensity (UHII) as indicator of urban heating. Atmospheric Research, v. 94, p. 491-500, 2009.

MIAO, S.; CHEN, F.; LEMONE, M.A.; TEWARI, M.; LI, Q.; WANG, Y. An observational and modeling study of characteristics of urban heat island and boundary layer structures in Beijing. Journal of Applied Meteorology and Climatology, v. 48, p. 484-501, 2009a.

PENG, S.; PIAO, S.; CIAIS, P.; FRIEDLINGSTEIN, P.; OTTLE, C.; BREON, F.; NAN, H.; ZHOU, L.; MYNENI, R.B. Surface Urban Heat Island Across 419 Global Big Cities. EnviromentalScience Technology, v. 46, p. 696-703, 2012.

ROQUE, W.V. Mapeamento geoambiental da área urbana de Manaus-AM. Dissertação (Mestrado em Geotecnia). Universidade de Brasília. Departamento de Engenharia Civil e Ambiental, Universidade de Brasília, 2006, 162 p.

SILVA, D.A. A influência das áreas verdes no clima da cidade de Manaus. Dissertação (Mestrado em Geografia). Universidade Federal do Amazonas. Manaus, Amazonas, 2009, $293 \mathrm{p}$.

SKAMAROCK, W.C.; KLEMP, J.B.; DUDHIA, J.; GILL, D.O.; BARKER, D.M.; WANG, W.; POWER, J.G. A description of the advanced research WRF version 2. NCAR/TN468+STR, 2005, 88 p

SOUZA, D.O. Influência da Ilha de calor urbana das cidades de Manaus e Belém sobre o microclima local. Tese (Doutorado em Meteorologia). Instituto Nacional de Pesquisas Espaciais, São José dos Campos, São Paulo, 2012, 203 p.

SOUZA, D.O.; ALVALÁ, R.C.S. Observational evidence of the urban heat of Manaus city, Brazil. Meteorological Applications, v. 21, p. 186-193, 2014.

SOUSA, S.B.; FERREIRA, L.G. Análise da temperatura de superfície em ambientes urbanos: um estudo por meio de sensoriamento remoto no município de Goiânia, Goiás (2002 - 2011). Confins [Online], 15 2012, posto online no dia 23 Junho 2012, consultado o 25 março 2015. URL: http://confins.revues.org/7631; DOI: 10.4000/confins.7631.

STREUTKER, D.R. A remote sensing study of the urban heat island of Houston, Texas. International Journal of Remote Sensing, v. 23, p. 2.595-2.608, 2002.

STWART, I.D. A systematic review and scientific critique of methodology in modern urban heat island literature.International Journal of Climatology, v. 31, p. 200-207, 2011.

TOMLINSON, C.J.; LEE, C.; THORNES, J.E.; BAKERA, C. Remote sensing land surface temperature for meteorology and climatology: a review. Meteorologial Applications, v. 18, p. 296-306, 2011.

UNFPA. The state of world population 2007: Unleashing the potential of urban growth. United Nations Population Fund, United Nations Publications 1 p., 2007.

WAN, Z. Collection-5 MODIS Land Surface Temperature Products Users' Guide. Institute for Computational Earth System Science. University of California, Santa Bárbara, 2007.

WAN, Z. New refinements and validation of the MODIS LandSurface Temperature/Emissivity products. Remote Sensing of Environment, v. 112, p. 59-74, 2008.

All the contents of this journal, except where otherwise noted, is licensed under a Creative Commons Attribution License CC-BY. 